\title{
TAMIZAJE Y TRATAMIENTO DE CHLAMYDIA TRACHOMATIS EN GESTANTES EN LIMA, PERÚ
}

\author{
Pedro García Aparcana ${ }^{1}$
}

\begin{abstract}
RESUMEN
Objetivos. El estudio tiene como objetivo implementar una prueba para la detección y tratamiento de clamidia durante la atención prenatal, determinando la aceptabilidad y la factibilidad de su tamizaje universal, así como medir la adherencia al tratamiento de la paciente y su pareja y además estimar la prevalencia de clamidia en gestantes. Materiales y métodos. Se realizó un estudio prospectivo en gestantes > 16 años, en 2 grandes hospitales en Lima (Instituto Nacional Materno Perinatal y Hospital Arzobispo Loayza) durante el año 2013. Se ofreció el tamizaje de clamidia a gestantes durante su primera atención prenatal utilizando hisopos vaginales auto-administrados (Aptima Combo 2 ensayo®, Hologic Gen-Probe). Se contactó con las pacientes positivas para Chlamydia en un plazo de 14 días, se les entregó consejería y el tratamiento gratuito directamente observado con 1 gramo de azitromicina por vía oral a ella y su pareja. Las gestantes que acudieron solas, se les proporcionó 1 gramo de azitromicina para llevar a casa para su pareja. Se realizó una prueba de cura 3 semanas después del tratamiento. Resultados. Se reclutó a 600 gestantes para el tamizaje. La edad promedio fue de $27,2 \pm 6,9$ años, la mediana de parejas sexuales en toda su vida fue $2,3 \pm 2,5$ parejas y una edad Gestacional media de $26,3 \pm 10,5$ semanas. La prevalencia de Chlamydia fue del 10\%(IC del 95\%: 7,7\% - 12,7\%). De las 60 pacientes positivas para clamidia, $59(98 \%)$ fueron tratadas. De las 59 pacientes tratadas, $52(88 \%)$ regresaron para la prueba de cura. Las 52 pacientes que aceptaron la prueba de cura fueron tratadas con éxito. Conclusión. La detección y tratamiento de Chlamydia en el embarazo fue factible y muy aceptado en los dos hospitales. La prevalencia de la infección por clamidia fue alta. Nuestros escenarios son óptimos para un ensayo clínico de detección universal y tratamiento oportunode clamidia y así prevenir los resultados adversos en el embarazo.
\end{abstract}

Palabras Clave: Tamizaje; Tratamiento; Clamidia; Gestantes (fuente: DeCS BIREME).

\section{SCREENING AND TREATMENT OF PREGNANT IN CHLAMYDIA TRACHOMATIS IN LIMA, PERU}

\begin{abstract}
Objectives. The study aims to implement a test for the detection and treatment of chlamydia during antenatal care, determining the acceptability and feasibility of universal screening and to measure adherence of the patient and her family and also to estimate the prevalence of chlamydia in pregnant women. Material and methods. A prospective study was conducted in pregnant women > 16 years, in 2 large hospitals in Lima ( Instituto Nacional Materno Perinatal and Loayza Archbishop Hospital) during 2013. Chlamydia screening is offered to pregnant women during their first prenatal care using self-administered vaginal swabs ( Aptima Combo 2 assay ${ }^{\circledR}$, Hologic Gen -Probe ). We contacted patients positive for Chlamydia within 14 days, they were given free counseling and directly observed treatment with azithromycin 1 gram oral route to her and her partner. The pregnant women attending alone, were given 1 gram of azithromycin to take home to your partner. A test of cure 3 weeks after the treatment was performed. Results. We recruited 600 pregnant women for screening. The average age was $27,2+6,9$ years, the medium of sexual partners in a lifetime partners was $2,3+2,5$ and a mean gestational age of $26,3+10,5$ weeks. Chlamydia prevalence was $10 \%$ ( $95 \% \mathrm{Cl}: 7,7 \%-12,7 \%)$. Of the 60 patients positive for chlamydia, 59 ( $98 \%$ ) were treated. Of the 59 patients treated, $52(88 \%)$ returned for test of cure. The 52 patients who accepted the test of cure were treated successfully. Conclusion. Chlamydia screening and treatment in pregnancy was feasible and highly accepted in both hospitals. The prevalence of chlamydia was high. Our scenarios are optimal for a clinical trial of universal screening and treatment oportunode chlamydia and prevent adverse pregnancy outcomes.
\end{abstract}

Key words: Screening;Treatment; Chlamydia; Pregnant women (source: MeSH NLM).

\section{INTRODUCCIÓN}

La Organización Mundial de la Salud (OMS) estima en su último informe que las tres infecciones de transmisión sexual (ITS) bacterianas de reporte más frecuente (sífilis, gonorrea y clamidiasis) entre sujetos entre los 15 y 49 años suman alrededor de 222 millones de casos nuevos anualmente en el mundo ${ }^{1}$ de ellos 105.7 millones corresponden a la más frecuente que es por Chlamydia trachomatis, manteniéndose esta infección sin cambio en la proporción mostrada en la revisión con la misma metodología en el año 2005; aún más, es la Región de las Américas la que tiene las más altas incidencia y prevalenciaestimadas en mujeres en todo el orbe (72.6 x 1000 y $7.6 \%$ respectivamente). El mantenimiento de estas cifras altas pueden deberse a múltiples factores, entre ellos el inicio más temprano de la actividad sexual, cambios sociales que derivan en mayor número de factores de riesgo para diseminar las ITS, y poco énfasis en la prevención y tratamiento de las parejas en

Médico Ginecoobstetra del Instituto Nacional Materno Perinatal. Director de la Oficina de Cooperación Científica Internacional del Instituto Nacional Materno Perinatal. Magister en Salud Pública Universidad de Washington-USA

Recibido: 10-10-13 Aprobado: 3-12-13 
forma conjunta. Todo ello obliga a preparar una serie de intervenciones que necesitan de un lobbing político y apoyo financiero para llevarse a cabo.

Mientras tanto esta infección que se comporta como una epidemia silenciosa con baja mortalidad pero muy alta morbilidad y costos sociales, continua su avance sigiloso produciendo lesiones muy frecuentemente irreversibles en el aparato genital femenino tales como la enfermedad inflamatoria pélvica, la cual deviene posteriormente en secuelas como el dolor pélvico crónico e infertilidad. Sin embargo, es durante la gestación cuando la infección clamidiasica presenta sus efectos agudos más devastadores, estando a la fecha asociada con aborto, parto prematuro, muerte fetal, bajo peso al nacer y puede ser transmitida verticalmente causando morbilidad y mortalidad neonatal ${ }^{2}$. Debido a que el diagnóstico de laboratorio para este microorganismo usualmente ha requerido procedimientos de alto costo o laboratorios especializados, la elección de las estrategias de control de estos agentes debe incluir el conocimiento de su magnitud en la población local y el desarrollo de pruebas que requieran tomas de muestra sencilla, de respuesta inmediata y de bajo costo ${ }^{3-4}$, tal como ya ha ocurrido por ejemplo con la sífilis.

Un primer objetivo del presente estudio por tanto es establecer la magnitud del problema en las gestantes. Además se establecerán algunos factores que pueden estar asociados a su aparición aunque con una importancia secundaria. Una segunda parte es ofrecer el tratamiento estándar más adecuado a la gestante que es la Azitromicina 1 gramo vía oral en dosis única evaluando su cumplimiento y la tasa de cura, es decir investigar operativamente la factibilidad de una intervención terapéutica en las gestantes y sus parejas, con la idea de reducir la reinfección de las mujeres y la diseminación a otras por parte de las parejas. De lograrse resultados positivos se podrá establecer la pertinencia de esta metodología en un futuro para su aplicación en otras instituciones de salud a nivel nacional.

\section{METODOLOGÍA}

Se realizó un estudio en dos fases. La primera como estudio transversal, respecto a la evaluación de la frecuencia de infección por Chlamydiatrachomatisen gestantes > 16 años, en 2 grandes hospitales en Lima (INMP y Hospital Loayza) y completar una entrevista breve para conocer aspectos epidemiológicosde la infección (edad, estado civil, grado de instrucción, paridad, edad gestacional, historia sexual, y síntomas de ITS en los últimos 12 meses). Para ello se ofreció el tamizaje de clamidia a gestantes durante su primera atención prenatal independientemente de la semana de gestación en la que se encontrara, utilizando hisopos vaginales auto-autoadministrados (AptimaCombo2ensayo ${ }$, HologicGen-Probe). Para ello existió un consentimiento informado y un asentimiento en el caso de menores de edad y se realizó una consejería preprueba.

Las muestras fueron procesadas en el Laboratorio de Infecciones de Transmisión Sexual de la Universidad Peruana Cayetano Heredia. Dos veces por semana se reportaban resultados, los cuales fueron colocados en las respectivas historias clínicas de las pacientes. En el caso de ser negativa, solo se colocó el informe en la historia habiéndosele advertido de ello a la paciente. En caso de ser positivo, el equipo de investigación la contactaba vía telefónica para acceder a una consejería post prueba, donde se iniciaba la parte experimental del estudio que consistía en medir la adherencia al tratamiento, la administración a la pareja y la tasa de cura lograda.

Se contactó a las pacientes positivas para Chlamydia en un plazo máximo de 14 días desde la toma de su muestra, y se les solicitó acudan con su pareja sexual al hospital para la consejería y administrarle tratamiento directamente observado con 1 gramo de azitromicina por vía oral. Las gestantes que acudieron sin su pareja recibieron consejería y tratamiento observado y se les proporcionó1 gramo de azitromicina para llevar a casa para su pareja. Finalmente se realizóuna pruebadecura 3 semanasdespués del tratamiento.

El análisis estadístico implico utilizar las medidas descriptivas para variables cuantitativas (media, desviación estándar) y cualitativas (porcentajes). Se formaron tablas de doble entrada y se calcularon los Odds Ratio con sus respectivos intervalos de confianza cuando fue pertinente o también se utilizó chi cuadrado y t student. El estudio conto con la aprobación del comité de ética del Instituto y del Hospital, así como de la Universidad Peruana Cayetano Heredia y University of California, Los Angeles.

\section{RESULTADOS}

Se estableció contacto con 640 gestantesy luego de explicar detalles y alcances del estudio se logró que 600 $(93,7 \%)$ firmen el consentimiento/asentimiento informado permitiendo empezar la toma de información y la muestra biológica correspondiente.

La edad promedio fue de $27,2 \pm 6,9$, sin embargo hubo una diferencia significativa entre las positivas y las negativas $(p<0,05)$.

El grado de instrucción no mostró ninguna tendencia significativa entre los grupos con /sin clamidiasis, sin embargo es de destacar que hasta un tercio de las pacientes entrevistadas refirieron educación superior, un cambio sustancial en el grado de instrucción de las pacientes que acuden a los hospitales públicos de Lima, El estado civil predominante fue el ser casada o conviviente, sin embargo hubo diferencias significativas 
entre los grupos positivos y negativos a la prueba, calculándose un OR de 2,31 (1,29-4,13) (Tabla 1).

Tabla 1. Características Demográficas según resultados del tamizaje para clamidiasis

\begin{tabular}{lccc}
\hline \multicolumn{1}{c}{ Características } & $\begin{array}{c}\text { Positivo } \\
(\mathrm{N}=60)\end{array}$ & $\begin{array}{c}\text { Negativo } \\
(\mathrm{N}=540)\end{array}$ & \multirow{2}{*}{$\mathrm{NS}$} \\
\cline { 2 - 3 } & $\mathrm{N}(\%)$ & $\mathrm{N}(\%)$ & \\
\hline Edad mediana (rango) & $23(20-38)$ & $27(22-33)$ & $\begin{array}{c}<0,05 \\
\text { (t-student) }\end{array}$ \\
\hline Educación & & & \\
Ninguno/Primaria/Secundaria & $41(68.3 \%)$ & $348(64.4 \%)$ & $\mathrm{OR}=1,19$ \\
Superior & $19(31.6 \%)$ & $192(35.5 \%)$ & $(0,67-2,10)$ \\
Estado civil & & & \\
Soltera/Separada/Viuda & $20(33.3 \%)$ & $96(17.7 \%)$ & $\mathrm{OR}=2,31$ \\
Casada/Conviviente & $40(66.7 \%)$ & $444(82.3 \%)$ & $(1,29-4,13)$ \\
\hline
\end{tabular}

Aproximadamente un $40-45 \%$ de las pacientes fueron primigestas y la distribución no se alteró al interior de los dos subgrupos en comparación.

La edad gestacional media fue de $26,3 \pm 10,5$ semanas. Más del $50 \%$ de todas estas pacientes llegaban después de las 28 semanas y la distribución por los grupos no mostro diferencias significativas.

La mediana de parejas sexuales en toda su vida fue $2,3 \pm 2,5$ no siendo diferente entre aquellas con infección versus aquellos que no la tenían. Lo mismo se encontró con el uso del preservativo.

Los síntomas de ITS no tuvieron correlato con el diagnóstico de laboratorio de infección por Chlamydia trachomatis. Cabe destacar la gran cantidad (más del $80 \%$ ) de gestantes con flujo vaginal (Tabla 2 ).

Tabla 2. Características obstétricas y comportamientos sexuales

\begin{tabular}{|c|c|c|c|}
\hline \multirow[t]{2}{*}{ Características } & $\begin{array}{c}\text { Positivo } \\
(\mathrm{N}=60)\end{array}$ & $\begin{array}{l}\text { Negativo } \\
(\mathrm{N}=540)\end{array}$ & \multirow[t]{2}{*}{ NS } \\
\hline & $\mathrm{N}(\%)$ & $\mathrm{N}(\%)$ & \\
\hline \multicolumn{4}{|l|}{ Paridad } \\
\hline Primer embarazo & $27(45 \%)$ & $191(35,4 \%)$ & $\mathrm{OR}=1,49$ \\
\hline Segundo embarazo o más & $33(55 \%)$ & $349(64,6 \%)$ & $(0,87-2,56)$ \\
\hline \multicolumn{4}{|l|}{$\begin{array}{l}\text { Edad gestacional } \\
\text { (en semanas) }\end{array}$} \\
\hline Primer trimestre $(1-12)$ & $11(18,3 \%)$ & $83(15,4)$ & \\
\hline Segundo trimestre $(13-27)$ & $15(25 \%)$ & $167(30,9)$ & $0,60(X 2)$ \\
\hline Tercer trimestre (28 a más) & $34(56,7 \%)$ & $290(53,7)$ & \\
\hline \multicolumn{4}{|l|}{ Historia Sexual } \\
\hline $\begin{array}{l}\text { Edad mediana de primera } \\
\text { relación sexual (rango) }\end{array}$ & $17(16-19)$ & $18(16-19)$ & $\begin{array}{c}0,10 \\
\text { (t-student) }\end{array}$ \\
\hline $\begin{array}{l}\text { Uso de condón en última } \\
\text { relación sexual }\end{array}$ & $3(5 \%)$ & $27(5 \%)$ & $0,95(X 2)$ \\
\hline \multicolumn{4}{|l|}{$\begin{array}{l}\text { Síntomas de ITS } \\
\text { (últimos } 12 \text { meses)* }\end{array}$} \\
\hline Descenso o flujo vaginal & $52(86,6 \%)$ & $472(87,4 \%)$ & $0,87(X 2)$ \\
\hline Verruga genital & $3(5 \%)$ & $33(6 \%)$ & $0,69(X 2)$ \\
\hline Llaga o herida genital & $2(3,3 \%)$ & $24(4,4 \%)$ & $0,73(X 2)$ \\
\hline
\end{tabular}

La prevalencia de Chlamydia fue del $10 \%$ (IC del $95 \%$ : $7,7 \%-12,7 \%)$.

De las 60 pacientes positivas para clamidia, 59 (98\%) fueron tratadas, 1 rechazo el tratamiento. De las 59 pacientes diagnosticadas y tratadas, solo $52(88 \%)$ regresaron para la prueba de cura a las tres semanas. Todas las pacientes que aceptaron la prueba de cura (52) fueron tratadas con éxito, sin embargo se tuvo que ofrecer retratamiento a seis de ellas, luego de lo cual todas estuvieron negativas, curadas.

Finalmente en cuanto a las parejas, 55 (93\%) fueron tratadas y en 4 casos (7\%) no aceptaron la terapia.

De los 55 varones que recibieron el tratamiento, 21 de ellos lo hizo mientras acompañaba a su pareja y 34lo hizo mediante la entrega por lo gestante en su casa del tratamiento. La medición de esta adherencia se hizo a través de la entrevista a la paciente luego de la prueba de cura, sin embargo para ellos no hubo prueba de cura.

\section{DISCUSIÓN}

Chlamydia trachomatis es la bacteria de transmisión sexual más frecuente en el mundo, con una mayor prevalencia en adolescentes y adultos jóvenes de ambos géneros. Dado que la infección en la mujer es hasta en $80 \%$ de los casos asintomática, constituye un factor de riesgo acumulativo de enfermedad inflamatoria pélvica (EIP) y de secuelas reproductivas.

Hasta hace unos años, se consideró incierto el papel de C. trachomatis sobre efectos adversos durante el embarazo. Actualmente, ha sido asociada con embarazo ectópico, aborto, parto prematuro, bajo peso al nacer, rotura prematura de membranasy mortalidad perinatal ${ }^{5-8}$. Recientemente, Haggerty y col. ${ }^{9}$ en un estudio de casos y controles anidado demostraron que la infección durante la gestación por Chlamydia trachomatis se asoció con 7.2 veces más probabilidad de presentar preeclampsia luego de ajustar para edad materna, índice de masa corporal, tabaquismo, raza e intervalo de tiempo entre la toma de muestras de sangre, abriendo una nueva posibilidad de resultado adverso por lo que es necesario mayores estudios prospectivos. El neonato adquiere la bacteria en su paso por el canal del parto y tiene riesgo de desarrollar conjuntivitis en $25-50 \%$ de los expuestos y hasta $17 \%$ de ellos desarrolla una neumonía ${ }^{10,11}$.Es probable que exista muchísima patología perinatal con origen en esta infección, todo esto resalta la trascendencia de estudiar esta patología y declararla como problema de salud pública.

Existe casi un consenso en la literatura que las cifras de pacientes infectadas por Chlamydia trachomatis está en ascenso permanente. Dicker y col indican que parte del aumento en la prevalencia de infecciones por 
C. trachomatis detectada en el mundo se debería a una mayor sensibilidad de las técnicas de diagnóstico actualmente en uso ${ }^{12}$ y no necesariamente a una mayor diseminación del germen.

Debido a todas estas potenciales complicaciones perinatales a partir de un estado infeccioso por Chlamydia trachomatis, y su aumento significativo en algunas regiones como la nuestra, donde al menos se mantiene en cifras elevadas permanentemente, la mayor parte de la literatura reciente recomienda un tamizaje universal en gestantes ${ }^{13}$. Nuestros hallazgos corroboran esta posición. Diez por ciento de prevalencia es una cifra alta que ameritaría una acción de salud pública nacional y nos atrevemos a plantear que dado este éxito de la intervención, puede resultar costo efectiva y requiere de un proyecto de mayor envergadura.

La literatura médica nacional informa de una frecuencia variable debido a las diferentes metodologías diagnósticas utilizadas y los momentos distintos en que fueron realizados. Así por ejemplo, en mujeres no gestantes León y col ${ }^{14}$ en el 2003 en la costa norte de nuestro país calculo una prevalencia de $14.9 \%$.La serie más grande nacionalen gestantes en la última década fue reportada por Hitti y col ${ }^{15}$ en el Instituto Nacional Materno Perinatal donde entre algo más de 1300 gestantes donde se buscaba la relación entre Mycoplasmagenitalium y parto pretérmino se encontró una prevalencia del $10 \%$ de clamidiasis utilizando PCR de secreción cervical, cifra que nuevamente hemos encontrado en esta oportunidad.

Respecto a la comparación con otros países de la región podemos mencionar por ejemplo que en el norte de Brasil, De Borborema-Alfaia y col. ${ }^{16}$ presento recientemente una pequeña casuística donde resalta la escasa información acerca de los resultados perinatales asociados a esta infección; demostraron una prevalencia materna de $11 \%$ mediante PCR e hicieron un seguimiento por 60 días de los neonatos de madres infectadas encontrando que $50 \%$ de los bebés hicieron síntomas respiratorios en dicho período de tiempo. Este último punto resalta la necesidad que la siguiente intervención se complemente con el seguimiento neonatal dada la importancia de la trasmisión vertical evidente a la luz de estas cifras. Pinto y col ${ }^{17}$ en Brasilea muestran $9.8 \%$ de prevalencia y hacen notar que la edad temprana, el inicio muy precoz de la actividad sexual y el número elevado de parejas sexuales son factores asociados y merecen alguna forma de intervención, pero que definitivamente también apoyan la universalidad del tamizaje en las gestantes. Aquí debemos de llamar la atención que, nuestras pacientes infectadas o no, no tuvieron diferencias en el número de parejas sexuales hasta el embarazo actual, porque es poco probable que existe un sesgo de respuesta, al ser entrevistas privadas.

Por otro lado en la región Metropolitana de Chile, Ovalle y col. ${ }^{18}$, señalan que la infección por Chlamydia es baja en el orden del $5.9 \%$ y que se muestra que en el lapso de los últimos años no ha incrementado significativamente. Chile es un país vecino que ha mostrado una baja prevalencia de esta ITS en forma longitudinal y muchas de sus instituciones no lo ven como una amenaza severa a la salud de sus mujeres, probablemente porque han adoptado medidas de salud pública mucho más contundentes.

Respecto a lo que sucede en otras latitudes, una investigación por Lavoué y col ${ }^{19}$ en Francia demostró que las mujeres asintomáticas que buscan una interrupción voluntaria del embarazo en la primera mitad del embarazo tuvieron una frecuencia de $6.7 \%$ de clamidiasis, estas mujeres fueron 2 veces más probable que sean menores de 30 años, que sean solteras, que tengan más de 11 semanas de embarazo y que no hayan usado anticoncepción, además fue 5 veces más frecuente entre aquellas con ninguno o un hijo previo. Es probable que el perfil de estas mujeres que buscaban una interrupción voluntaria del embarazo sea un poco diferente al promedio de la población femenina y por eso las cifras de riesgos altas. Por otro lado en la última década en los EUA, en clínicas de atención prenatal de tipo público la prevalencia encontrada fue de $8.3 \%{ }^{20}$, lo que los coloca en una situación conflictiva y más aún si la mayoría de sus reportes señalan un incremento progresivo. Una situación muy diferente es planteada para otras latitudes como por ejemplo al sur de la India donde la prevalencia encontrada fue del $0.1 \%{ }^{21}$, y como es lógico los autores no recomiendan el tamizaje universal.

Como se puede entender a partir de estas referencias, el tamizaje universal dependerá su instauración según el nivel de prevalencia detectado. Los expertos en salud pública señalan que cuando es mayor del $3 \%$, la relación costo-efectividad es buena y empieza a ser mandatorio. Por tanto para los centros de salud estudiados, es más que suficiente la cifra encontrada como argumento para implementar un sistema de tamizaje universal.

Sin embargo, dadas algunas publicaciones recientes, la pregunta que surge es cuál es el momento o momentos para hacer el tamizaje. Hood y Nerhood 22 demostraron mediante un estudio longitudinal como un grupo de gestantes diagnosticadas y tratadas en el primer trimestre se mantuvieron negativas luego del tratamiento, pero en cambio hubo un subgrupo que dio positividad por vez primera después de las 34 semanas, por lo que cada trimestre tiene una independencia de resultados y cuestiona si solo es necesario el tamizaje en un punto en la gestación. Finalmente si así fuera, el tamizaje tardío podría ser importante en reducir los resultados adversos neonatales tempranos. Para fines prácticos y por ser una propuesta que deberá lidiar con patrones culturales y logísticos bastante duros, el tamizaje que se propone creemos debe continuar siendo en el momento que la paciente llega a la Institución, siempre y cuando el tratamiento de la pareja tenga un éxito alto. 
Otro aspecto es, que método y que tipo de muestra biológica puede utilizarse. En nuestro estudio se utilizó una técnica que tiene cerca del $98 \%$ de especificidad y $97 \%$ de sensibilidad por lo tanto muy confiable. Por otro lado la muestra de secreción vaginal y no necesariamente la cervical es suficiente (lo que permite un auto muestreo), y en cuanto a la orina ha demostrado queno da mejores resultados ${ }^{23}$

Un problema colateral es la asociación entre infección por VIH y una ITS estableciéndose una relación de potenciación en su ocurrencia. Asavapiriyanonty col. ${ }^{24}$ este año en Tailandia demuestran que las mujeres infectadas con VIH tienen una frecuencia mucho más alta de ITS pero lo fueron especialmente aquellas que estaban embarazadas, así por ejemplo la clamidiasis en estas mujeres que viven con el VIH conto para el $17.7 \%$ de las gestantes versus $6.6 \%$ para aquellas no embarazadas, mientras que en el mismo país la prevalencia histórica en la población no infectada por $\mathrm{VIH}$ ha sido $9 \%$. Es importante no perder de vista esta relación para que cada vez que se reporte una paciente con Clamidiasis se hagan todos los esfuerzos por demostrar la ausencia o presencia de otras ITS.

Aunque el tamizaje para la infección por Chlamydiatrachomatis no está establecido por guías nacionales lo mismo que para otros países de la región, aun en aquellos que han establecido su universalidad las evaluaciones de sus programas demuestran una distancia amplia con lo optimó básicamente por incumplimiento del personal médico, así por ejemplo Li y col. ${ }^{25}$ reportan una baja captación para tamizaje en Australia. Esto debe de llevar a la meditación y a un planeamiento estratégico muy bien diseñado para que el personal de salud integre a sus actividades esta metódica.

El momento que es oportuno para diagnosticar y tratar la infección por Chlamydiatrachomatis en la gestación siempre será cualquiera. Todas las veces que se pueda contar con algún método diagnóstico será una oportunidad perdida menos. Podrá ser preferencialmente durante la atención prenatal, pero debería de tomarse en cuenta otras oportunidades que las pacientes llegan buscando atención como son las emergencias obstétricas. Krivochenitsery col. ${ }^{26}$ demostraron que durante las atenciones en la Emergencia de 179 gestantes que acudieron por diversas patologías intercurrentes del embarazo y que fueron positivas en el tamizaje de clamidiasis, $80 \%$ de ellas tuvieron diagnóstico clínico de cervicitis y $71 \%$ presentaron dolor abdominal, ninguna de ellas fue tratada, posteriormente y como parte del estudio $80 \%$ de ellas lograron ser contactadas y tratadas, y adicionalmente un $20 \%$ fueron perdidas en el seguimiento. Es muy importante evitar la reinfección y para ello es crítico dar tratamiento a la pareja. Con el fin de maximizar dicho enfoque algunos autores ${ }^{27-29}$ han señalado varias estrategias para mejorar la entrega de tratamiento a la pareja, como por ejemplo citar a la pareja mediante aviso por medio de la gestante, o mediante la llamada directa del personal que atiende el caso a la pareja, entrega del tratamiento a la gestante para que se lo entregue a su pareja, entre otros. Todas ellas han sido parte de esta intervención y el resultado creemos que ha sido muy bueno.

Si no se hubiera realizado esta investigación cerca de 60 gestantes hubieran continuado viviendo con esta infección, es posible que hubieran ocurrido muchas complicaciones perinatales y eventualmente las parejas o ellas mismas podrían haber diseminado aún más el germen a otros individuos.

En conclusión podemos decir que la prevalencia de la infección por clamidia fue altapara la realidad de los dos hospitales estudiados, y que este hallazgo se logró por la gran aceptabilidad de los pacientes y el personal involucrado, lo que permite enfatizar la factibilidad de su implementación a gran escala.

Nuestros escenarios son óptimos para un ensayo clínico sistemático de detección y tratamiento de clamidia y con ello prevenir resultados adversos en el embarazo, que deben ser incluidos como variables resultado en una próxima intervención.

\section{REFERENCIAS BIBLIOGRÁFICAS}

1. Global incidence and prevalence of selected curable sexually transmitted infections - 2008. World Health Organization 2012. http://apps.who.int/iris/ bitstream/10665/75181/1/9789241503839_eng.pdf

2. Mullick S, Watson-Jones D, Beksinska M, Mabey D. Sexually transmitted infections in pregnancy: prevalence, impact on pregnancy outcomes, and approach to treatment in developing countries. Sex Transmlnfect 2005; 81: 294-302

3. Centers for Disease Control and Prevention. Sexually transmitted diseases treatment guidelines, 2010. MMWR Morb Mortal WklyRep 2010/59 (RR12): 1-114.

4. Jani IV. The need for systematic evaluations of diagnostic tests. ClinInfectDis 2010; 51: 609-10

5. Blas M M, Canchihuaman FA, Alva I E, Hawes S E. Pregnancy outcomes in women infected with Chlamydia trachomatis: a population-based cohort study in Washington State. Sex TransmInfect 2007; 83: 314-8.

6. Rours G I, Duijts L, Moll H A, Arends L R, de Groot R, Jaddoe $\mathrm{V}$ W, et al. Chlamydia trachomatis infection during pregnancy associated with preterm delivery: a populationbased prospective cohort study. Eur J Epidemiol 2011; 26: 493-502

7. Chojnacka K, Szczapa J, Kedzia W. Perinatal transmission of Chlamydia trachomatis and its complication in preterm infants.Ginekol Pol. 2012 Feb;83(2):116-21.

8. Silva MJ, Florêncio GL, Gabiatti JR, Amaral RL, EleutérioJúnior J, Gonçalves AK. Perinatal morbidity and mortality associated with chlamydial infection: a meta-analysis study.Braz J InfectDis. 2011 Nov-Dec;15(6):533-9.

9. Haggerty CL, Klebanoff MA, Panum I, Uldum SA, Bass DC, Olsen J, Roberts JM, Ness RB. Prenatal Chlamydia trachomatis infection increases the risk of preeclampsia. Pregnancy Hypertens.2013 Jul 1;3(3):151-154. 
10. Valencia C, Prado V, Ríos M, Cruz MA, Pilorget J J.Prevalencia de Chlamydia trachomatis en conjuntivitis neonatal determinada mediante las técnicas de inmunofluorescencia y amplificación génica. RevMed Chile 2000; 128: 758-65.

11. Martínez MA, Millán F, González C. Chlamydia trachomatis genotypes associated with pneumonia in Chilean infants. Scand J InfectDis 2009; 41: 313-6

12. Dicker L W, Mosure D J, Levine W C, Black C M, Berman S M. Impact of switching laboratory tests on reported trends in Chlamydia trachomatis infections. Am J Epidemiol 2000; 151: 430-5.

13. Mishori R, McClaskey EL, WinklerPrins VJ. Chlamydia trachomatis infections: screening, diagnosis, and management. AmFam Physician.2012 Dec 15;86(12):1127-32

14. León SR, Konda KA, Klausner JD, Jones FR, Cáceres CF, Coates TJ; NIMH Collaborative HIV/STD Prevention Trial Group. Chlamydia trachomatis infection and associated risk factors in a low-income marginalized urban population in coastal Peru.RevPanam Salud Publica. 2009 Jul;26(1):39-45.

15. Hitti J, Garcia P, Totten P, Paul K, Astete S, Holmes KK. Correlates of cervical Mycoplasma genitalium and risk of preterm birth among Peruvian women.Sex TransmDis. 2010 Feb;37(2):81-5.

16. De Borborema-Alfaia AP, de Lima Freitas NS, Filho SA, Borborema-Santos CM. Chlamydia trachomatis infection in a sample of northern Brazilian pregnant women: prevalence and prenatal importance.Braz J InfectDis. 2013 SepOct;17(5):545-50.

17. Pinto VM, Szwarcwald CL, Baroni C, Stringari LL, Inocêncio LA, Miranda AE. Chlamydia trachomatis prevalence and risk behaviors in parturient women aged 15 to 24 in Brazil.Sex TransmDis. 2011 Oct;38(10):957-61.

18. Ovalle A, Martínez MA, de la Fuente F, Falcon N, Feliú F, Fuentealba F, Gianini R. Prevalence of sexually transmitted infections in pregnant women attending a public hospital in Chile. Rev Chilena Infectol. 2012 Oct;29(5):517-20

19. Lavoué V, Vandenbroucke L, Lorand S, Pincemin P, Bauville E, Boyer L, Martin-Meriadec D, Minet J, Poulain P, Morcel K. Screening for Chlamydia trachomatis using self-collected vaginal swabs at a public pregnancy termination clinic in France: results of a screen-and-treat policy.Sex TransmDis. 2012 Aug;39(8):622-7
20. Satterwhite CL, Gray AM, Berman S, Weinstock H, Kleinbaum D, Howards PP. Chlamydia trachomatis infections among women attending prenatal clinics: United States, 2004-2009. Sex TransmDis. 2012 Jun;39(6):416-20

21. Vidwan NK, Regi A, Steinhoff M, Huppert JS, Staat MA, Dodd C, Nongrum R, Anandan S, Verghese V. Low prevalence of Chlamydia trachomatis infection in non-urban pregnant women in Vellore, S. India.PLoSOne. 2012;7(5):e34794

22. Hood EE, Nerhood RC. The utility of screening for chlamydia at 34-36 weeks gestation.W V Med J. 2010 SepOct;106(6):10-1.

23. Roberts SW, Sheffield JS, Mclntire DD, Alexander JM. Urine screening for Chlamydia trachomatis during pregnancy. Obstet Gynecol. 2011 Apr;117(4):883-5

24. Asavapiriyanont $S$, Lolekha R, Roongpisuthipong $A$, Wiratchai A, Kaoiean S, Suksripanich O, Chalermchockcharoenkit A, Ausavapipit J, Srifeungfung S, Pattanasin S, Katz KA. Sexually transmitted infections among HIV-infected women in Thailand.BMC Public Health. 2013 Apr 22;13( 1):373.

25. Li Z, Chen M, Guy R, Wand H, Oats J, Sullivan EA. Chlamydia screening in pregnancy in Australia: integration of national guidelines into clinical practice and policy.Aust $\mathrm{N} Z \mathrm{~J}$ ObstetGynaecol. 2013 Aug;53(4):338-46.

26. Krivochenitser R, Jones JS, Whalen D, Gardiner C. Underrecognition of cervical Neisseria gonorrhoeae and Chlamydia trachomatis infections in pregnant patients in the ED.Am J Emerg Med. 2013 Apr;31(4):661-3.

27. Fitzmaurice E, Keller E, Trebbin J, Wilson J. Strategies for partner management when treating sexually transmitted infection.J Midwifery Womens Health.2011 NovDec;56(6):608-14.

28. Weisz J, Lozyniak S, Lane SD, Silverman R, DeMott K Wojtowycz MA, Aubry RH, Koumans EH. It takes at least two: male partner factors, racial/ethnic disparity, and Chlamydia trachomatis among pregnant women.J Health Care Poor Underserved. 2011 Aug;22(3):871-85

29. Cameron ST, Glasier A, Scott G, Young H, Melvin L, Johnstone $A$, Elton $R$. Novel interventions to reduce reinfection in women with chlamydia: a randomized controlled trial.Hum Reprod. 2009 Apr;24(4):888-95 